\title{
Description of the larva and pupa of Apion brevicorne Gerstaecker, 1854 (Coleoptera: Brentidae: Apioninae) with biological information
}

\author{
Rosana Maria de Lima ${ }^{1,3}$; Sandreli Berenice de Oliveira Santos ${ }^{1,4}$; Stephanie Vaz ; \\ Ana Laura Soares Gomes ${ }^{1,5}$ \& Wesley Oliveira de Sousa ${ }^{1,6}$ \\ 1 Universidade Federal de Rondonópolis (UFR), Departamento de Biologia. Rondonópolis, MT, Brasil. \\ 2 Universidade Federal do Rio de Janeiro (UFRJ), Instituto de Biologia (IB), Departamento de Ecologia. Rio de Janeiro, RJ, Brasil. \\ ORCID: http://orcid.org/0000-0002-2616-640X.E-mail: anievaz@gmail.com \\ ${ }^{3}$ ORCID: http://orcid.org/0000-0002-0076-6909. E-mail: rosana.lima0203@gmail.com \\ ${ }^{4}$ ORCID: http://orcid.org/0000-0001-6788-8209. E-mail: sandy.bi02016@gmail.com \\ ${ }^{5}$ ORCID: http://orcid.org/0000-0002-8554-5522. E-mail: sg.analaura@gmail.com \\ ${ }^{6}$ ORCID: http://orcid.org/0000-0002-5564-1759. E-mail: entomoi@hotmail.com
}

\begin{abstract}
Apion brevicorne Gerstaecker, 1854 (Coleoptera: Brentidae: Apioninae) is an ecologically and economically important weevil that feeds on seeds and tissues of trees in the genus Copaifera L. (Fabaceae). Although the genus Apion comprises 16 species restricted to the Paleartic region, the Neotropical species $A$. brevicorne is still considered as incertae sedis due to the absence of a systematic study about it. The first descriptions and illustrations of Apion brevicorne are provided here. Diagnostic characters of larva and pupa are included and compared with 13 species from other biogeographic regions. Details of immature Apioninae species associated with host plants from the Neotropical region are described for the first time.
\end{abstract}

Key-Words. Apionini; Cerrado; Copaifera malmei; South America; Trichapiina.

\section{INTRODUCTION}

The subfamily Apioninae (Brentidae) (Kuschel, 1995; Wanat, 2001; Marvaldi et al., 2002; AlonsoZarazaga \& Wanat, 2014) comprises often very small beetles $(0.75-13.00 \mathrm{~mm}$ long; more than $95 \%$ known species is less than $3 \mathrm{~mm}$ ). Adults tend to feed on the foliage of the larval host plant. Larvae feed on a variety of plant parts, including roots, inflorescences, seeds, and other tissues where they are endophagous and often form galls (Alonso-Zarazaga \& Wanat, 2014; De Sousa et al., 2019). The most diverse group of apionines (supertribe Apionitae) feed on at least 23 families (15 orders) of angiosperms, including the widespread plant family Fabaceae (Anderson \& Kissinger, 2002; Badenes-Perez \& Jhonson, 2007; Lima et al., 2008; Maia, 2012; Alonso-Zarazaga \& Wanat, 2014). With a total of around 2,200 described species (Alonso-Zarazaga \& Wanat, 2014), they are reasonably well-known elsewhere, while in the Neotropical Region the Apioninae and their host associations are poorly known (Alonso-Zarazaga \& Wanat, 2014).
Apionine species can be found from high altitudes to sea level wherever they occur (Anderson \& Kissinger, 2002; Alonso-Zarazaga, 2004; Oberprieler et al., 2007; Alonso-Zarazaga \& Wanat, 2014). The Apioninae includes about 205 genera and subgenera (Alonso-Zarazaga \& Lyal, 1999; Alonso-Zarazaga \& Wanat, 2014), seven supertribes and nine tribes (Alonso-Zarazaga, 1990; Bouchard et al., 2011; De Sousa et al., 2019). The supertribes Antliarhinitae, Cybebitae, Mecolenitae, Myrmacicelitae and Tanaitae comprise less than 60 species in Africa and the Australo-Pacific region. The Rhadinocybitae (> 400 species in ca. 40 genera) are found throughout the Australo-Pacific region. The Apionitae is the largest supertribe (with five tribes) and includes ca. $90 \%$ of all Apioninae species. The Apionini is the most diverse tribe with 15 subtribes and ca. 1,350 species (Kuschel, 1995; Wanat, 2001; Bouchard et al., 2011; AlonsoZarazaga \& Wanat, 2014; Winter et al., 2017; De Sousa et al., 2019).

In the Neotropical region, all 400 species (190 in South America) belong in the Apionitae with 22 genera (19 in South American) and three 
tribes. The Apionini includes 10 genera (seven from South America) and the monotypic South American Chilapiini and Noterapiini. Ten genera remain incertae sedis within the Apionitae (Kissinger, 1968, 2002, 2003, 2005a, 2005b; Wibmer \& O'Brien, 1986; AlonsoZarazaga, 2004; De Sousa et al., 2019). A recent, updated, classification of the Apioninae in Brazil includes 10 genera, two new species, and a new record, for a current total of 89 species (De Sousa \& Ribeiro-Costa, 2018; De Sousa, et al., 2019). Fifty-three species remain incertae sedis, including Apion brevicorne Gerstaecker, 1854 (see De Sousa et al., 2019). We assume that this species most likely does not belong in Apion, which is monophyletic and includes 16 species restricted to the Palearctic region (Alonso-Zarazaga, 1990). However, in the absence of a systematic study for the species, we are not sure which genus to assign it to. Thus, for the purpose of the immatures descriptions, this species is kept being regarded as Apion brevicorne.

Adult of Apioninae is reasonably well described, while the immature stages remain poorly known in much of the world (Emden, 1938; Wang et al., 2013) but are completely unknown in the South America region. Some small amount of information on larvae is available for ca. 40 species outside of the Neotropical Region, much of which includes only simple descriptions for larvae identification in keys. Of the 35 species in the Palearctic, 23 are in one key for larvae identification (Emden, 1938), while larvae of 12 other species have been described in detail: Malvapion malvae (Fabricius, 1775) (Malvapiina), Pirapion immune (Kirby, 1808) (Oxystomatina) (Williams, 1968), six Exapion Bedel species (Exapiina) (May, 1994; Sanz Benito \& Gurrea Sanz, 1999) also with description of pupae, and only larvae are described in Apion frumentarium (Linnaeus, 1758) (Apionina), Pseudaplemonus aeneicollis (Gerstaecker, 1854) (Aplemonina) and Stenopterapion margelanicum (Wagner, 1912) (Oxystomatina) (Nikulina, 2016), and both larvae and pupae of Squamapion elongatum (Germar, 1817) (Kalcapiina) (Letowski et al., 2015). Larvae and pupae in the Palearctic and Oriental Pseudaspidapion botanicum Alonso Zarazaga \& Wang, 2011 (Aspidapiina) are also described in detail (Wang et al., 2013). The Nearctic region includes five species in three genera that were briefly treated in a single key for larval identification: Coelocephalapion segnipes (Say, 1831), C. subornatum (Fall, 1898) (Oxystomatina), Fallapion ellipticum (Smith, 1884) (Piezotrachelina), Trichapion griseum (Smith, 1884) and T. rostrum (Say, 1826) (Trichapiina) (Emden, 1938).

Because Apion brevicorne is an important fruit and seed predator of Copaifera spp., here we describe and illustrate in detail the external morphology of their last larval instar and their pupa. This is the first description of immature stages in the Neotropical Apioninae, and this information is fundamental for future studies of the biology, ecology, taxonomy and systematics of weevils in the Neotropical region, and for providing foundations for a future re-assessment of the Neotropical species currently classified in Apion.

\section{MATERIAL AND METHODS}

The larvae and pupa were collected individually by hand, from April through September 2018, from seed pods of Copaifera malmei Harms, in the Cerrado (Brazilian savanna) at the Universidade Federal de Rondonópolis (UFR, $16^{\circ} 46^{\prime} \mathrm{S}, 54^{\circ} 58^{\prime} \mathrm{W}$ ). Seed pods were dissected to find larvae and pupae in the Water Analysis and Applied Ecology Laboratory (LAHEA/UFR). About 50 larvae and 50 pupae were given in hot water and then set in $70 \%$ alcohol and deposited in the Entomological Reference Collection of the Department of Biology (DBFR) for morphological study.

Larvae, their mouthparts, and pupae were drawn using a Zeiss Stemi 200-C stereomicroscope (50x) and Nikon Eclipse E200 microscope coupled to a camera lucida (40X). Larvae were decapitated, and the head was cleared in a $10 \%$ potassium hydroxide $(\mathrm{KOH})$ solution and then rinsed in water. The head capsule and all mouthparts were first examined using a Zeiss Stemi 200-C stereomicroscope, then mounted on slides or placed in depression slides containing glycerin (May, 1994), and subsequently examined under optical Nikon Eclipse E200 microscope coupled to a camera lucida. Illustrations were made by a line drawing CorelDRAW X5 program. Specimens were later examined using stereo- and compound light microscopes and scanning electron microscopy (SEM). For the latter, specimens were first dehydrated in a series of progressively increasing concentrations of ethanol (70-100\%) and then critical-point dried. Specimens were then mounted on stubs, sputter coated with $35 \mathrm{~nm}$ of gold and examined using a JEOL JSM-6390LV scanning electron microscope. The material was photographed at the Optical and Scanning Microscopy Image Laboratory at the Universidade Federal do Rio de Janeiro (UFRJ), Rio de Janeiro, Brazil.

Morphological characters were measured by using a BEL Photonics stereomicroscope with Optical Microscope Imaging Software. Larval measurements (reported in $\mathrm{mm}$ ) included body length (with head, as distance between apical margin of head capsule and dorsal margin of the tenth abdominal segment) in lateral view ( $N=20$ individuals); body width (at metathorax, $\mathrm{N}=10$ ); width of head capsule in front view $(\mathrm{N}=10)$. Pupal (10 individuals) measurements included length (lateral view) and width (dorsal view). The number of setae and bilateral structures were counted on one side of the body. Terminology, abbreviations and chaetotaxy of both larvae and pupae followed Alonso-Zarazaga \& Wanat (2014) and Oberprieler et al. (2014). Suprageneric classification follows Bouchard et al. (2011).

Larval and pupal chaetotaxy of Apion brevicorne were compared with 13 species in the Apioninae: Malvapion malvae (Malvapiina), Pirapion immune (Oxystomatina) (Williams, 1968), six Exapion species (Exapiina) (May, 1994; Sanz Benito \& Gurrea Sanz, 1999), Apion frumentarium (Apionina), Pseudaplemonus aeneicollis (Aplemonina) and Stenopterapion margelanicum (Oxystomatina) (Nikulina, 2016), Squamapion elongatum (Kalcapiina) (Letowski et al., 2015) and Pseudaspidapion botanicum (Aspidapiina) (Wang et al., 2013). 
The identification of adults in this study was confirmed by comparison with a specimen of Apion brevicorne identified by David G. Kissinger (label data: 11.308; Esc. Nac. Agr., Brasil Central: IX-1945, C. de Araujo; 98; Apion brevicorne Gerst. det. Kissinger) on loan to us by the Museu de Zoologia, Universidade de São Paulo, São Paulo (MZSP). Adult male and female images and drawings of the male genitalia allowed the identification of $A$. brevicorne. We provided five adult specimens of each sex to the following Brazilian institutions: Coleção Entomológica Pe. J.S. Moure, Departamento de Zoologia, Universidade Federal do Paraná, Curitiba (DZUP); Coleção Entomológica do Instituto de Biociências, Universidade Federal de Mato Grosso, Cuiabá (UFMT); Instituto Nacional de Pesquisas da Amazônia, Manaus (INPA);
Museu Nacional, Universidade Federal do Rio de Janeiro, Rio de Janeiro (MNRJ); Museu de Zoologia, Universidade de São Paulo, São Paulo (MZSP).

\section{RESULTS}

\section{Apion brevicorne Gerstaecker, 1854}

(Figs. 1-22; 29-35)

Material examined: Brazil. Mato Grosso: Rondonópolis, Campus da Universidade Federal de Rondonópolis (UFR), April to September 2018, host plant (Copaifera malmei Harms), 50 larvae (10 dissected, 9 metalized for SEM); 50 pupae, (9 metalized for SEM) (DBFR).
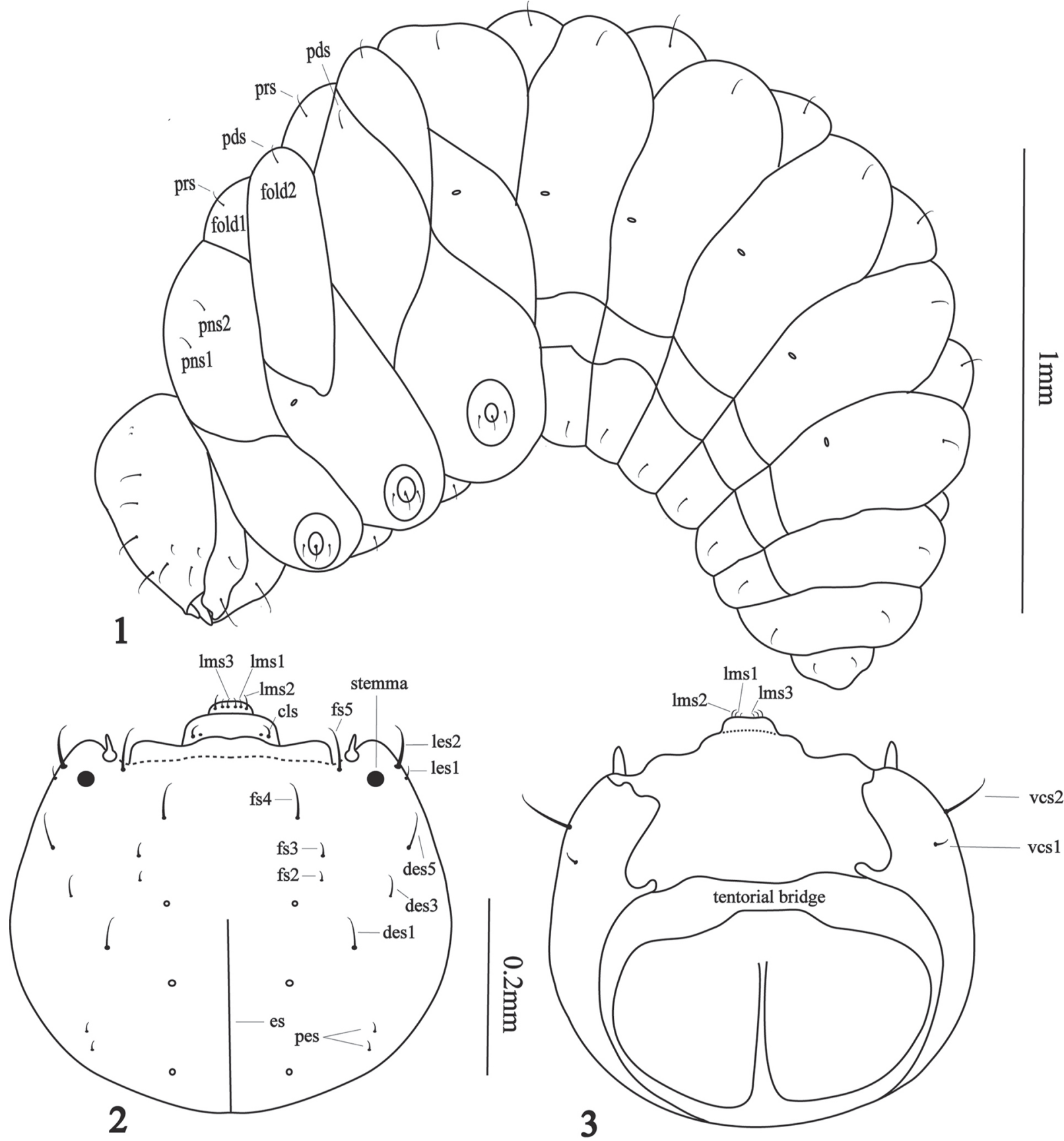

Figures 1-3. Apion brevicorne Gerstaecker, last instar larva: (1) habitus, lateral; (2) head capsule, frontal; (3) head capsule, ventral. Abbreviations: [seta (ae)-s.]: des = dorsal epicranial s.; es = epicranial suture; $f s=$ frontal epicranial s.; les = lateral epicranial s.; pes = posterior epicranial s.; $p n s=$ pronotal s.; prs $=$ prodorsal s.; $v c s=$ ventral epicranialss. 
Mature larva description (Figs. 1, 10): Measurements (in $\mathrm{mm}$ ): body length: 2.11-2.82, body width (metathorax): 1.30-1.50, head width: 0.66-0.76.

General: Body robust, sub-circular in cross-section, strongly dorso-ventrally curved (C-shaped); cuticle minutely spiculate, without visible pigmentation, areas sclerotized and devoid of roughness.

Colouration: Body distinctly white with copious white fat within; head capsule yellowish to pale brown.

Head (Figs. 2-3, 11): Slightly sclerotized; clypeus slightly darkened at the base, darkened labrum; epicranial line narrow with endocarina as an extension; frontal lines indistinct. Epicranium with 2 lateral setae (les), les 1 shorter than les2. Dorsal epicranium with 3 setae (des 1, 3 and 5). Posterior epicranium with 2 tiny setae (pes). Frons with 4 setae $\left(f_{s}\right), f_{s} 2$ and $f_{s} 3$ very short, $f_{s} 4$ medial and $f_{s} 5$ laterally positioned close to antennae. Ventral epicranium with 2 ventral setae (vcs), near les. Postoccipital condyles absent; tentorial bridge without anterior and posterior projections; hypopharyngeal bracon absent. Clypeus transverse, bearing 1 seta $(\mathrm{c} / \mathrm{s})$, inner side of cls bearing 1 sensilla. Antenna (Figs. 13-14) one-segmented, with rodlike accessory sensory appendage (acap) more than $3 \times$ as long as wide, with 2 spinose projections and 1 sensillum. Stemma (ocular spots) at the base of the antenna.
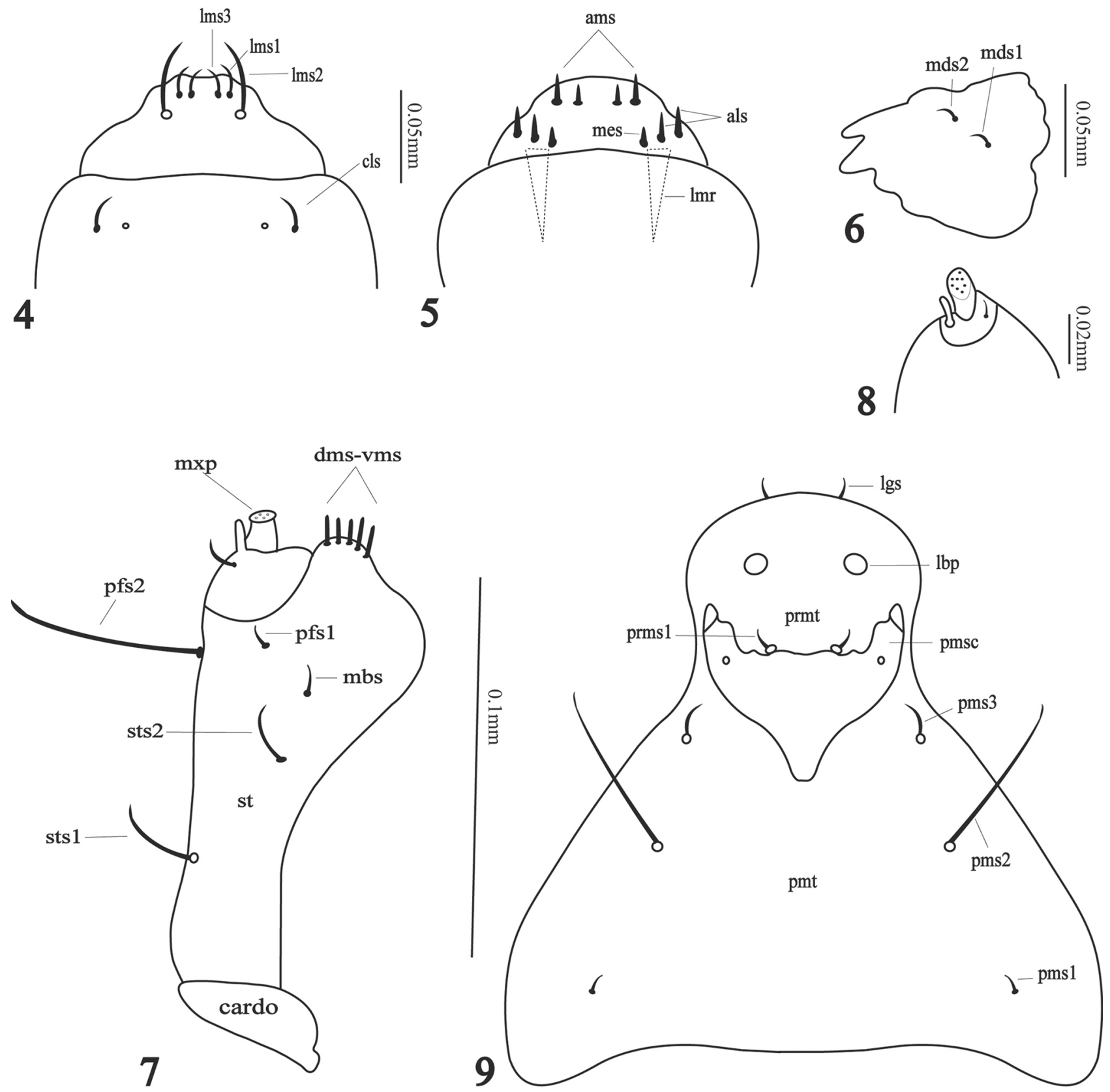

Figures 4-9. Apion brevicorne Gerstaecker, mouthparts: (4) clypeus and labrum, dorsal; (5) epipharynx; (6) mandible, dorsal; (7) maxilla; (8) maxillary palp; (9) labium.

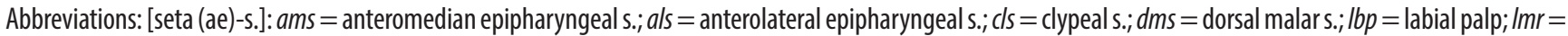
labral rods; Igs = ligular s.; Ims = labral s.; $m b s=$ basioventral seta s.; $m e s=$ median epipharyngeal s.; $m d s=$ mandibular s.; $m x p=$ maxillary palp; $p m t=$ postmentum; $p m s=$ postmental s.; $p r m t=$ prementum; $p r m s=$ premental s.; $p m s c=$ premental sclerite; $p f s=$ palpiferal s.; $s t=s t i p e s ; s t s=s t i p i t a l ~ s . ; v m s=v e n t r a l ~ m a l a r ~ s$. 
Mouthparts (Figs. 4-9, 12): Labrum (Fig. 4) subtrapezoidal with lateral margins sinuose, with 3 setae (Ims), Ims2 three times longer than two remining setae. Epipharynx (Fig. 5) with 2 long, stout lateral rods (ImR), 2 anterolateral setae (als), 2 anteromedian setae (ams), and 1 median seta (mes); epipharyngeal setae stout, short and apically rounded, lacking epipharyngeal sensory pores between pairs of ImR. Mandibles (Fig. 6) symmetrical, apically bidentate, length and width approximately equal, cutting edge with 1 small, but evident, rounded tooth; laterodorsal surface with 2 mandibular setae (mds 1-2), without sensilla. Maxilla (Fig. 7): palpifer indistinct; maxillary palpi (MxP, Figs. 7-8) with 2 segments, basal segment with 1 long, basal accessory process, 1 short seta, apical segment cylindrical, apically flattened with dense crenulate setae; mala with 5 stout, short, indistinguishable setae dms-vms; stipes (st) with 2 stipital setae (sts 1-2), 1 basioventral seta (mbs) and 2 palpiferal setae (pfs 1-2),

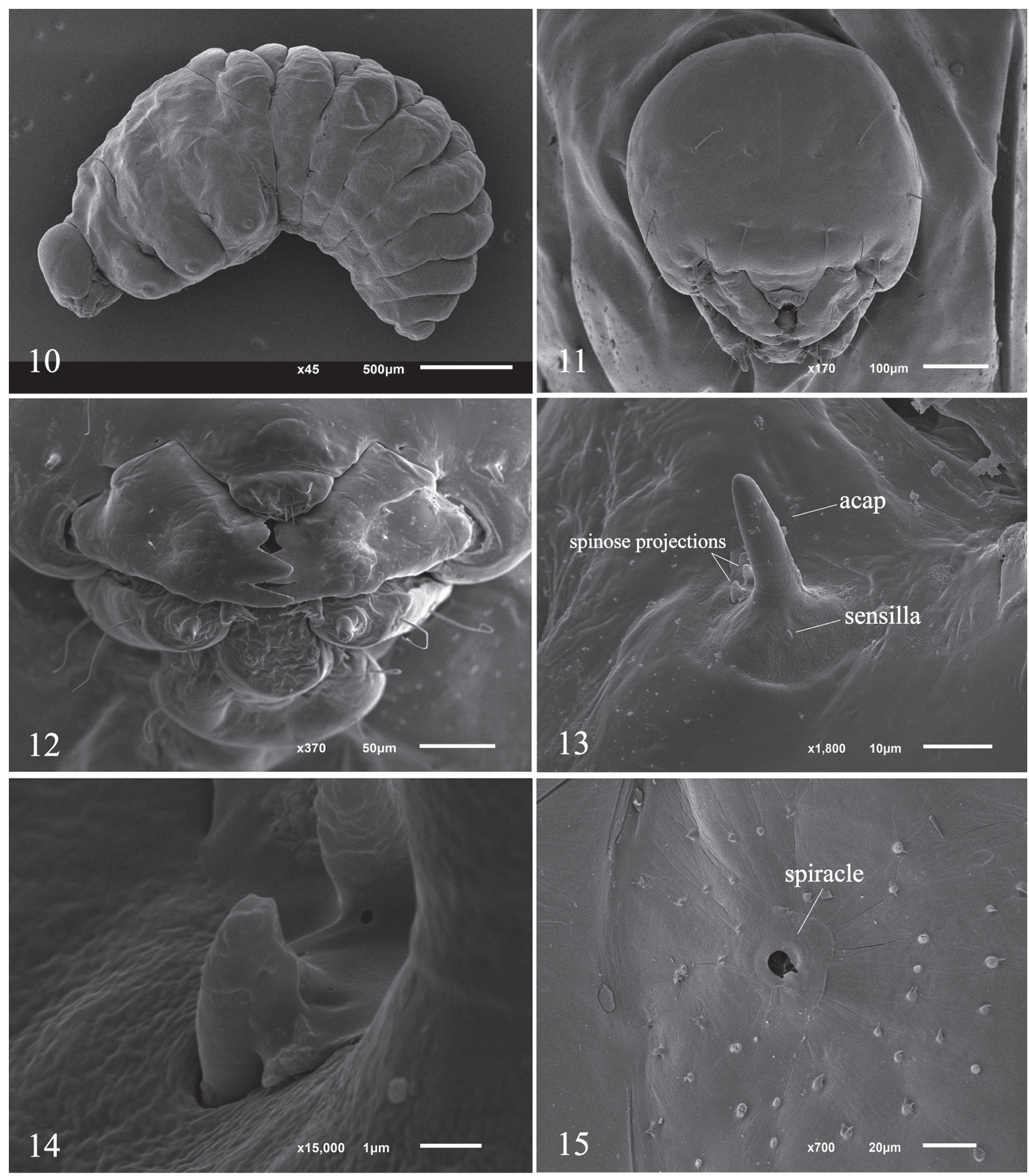

Figures 10-15. Apion brevicorne Gerstaecker, SEM micrographs larva: (10) habitus, lateral; (11) head, frontal; (12) mouthparts, frontal; (13-14) antenna; (15) spiracle. Legend: Acap = accessory sensory appendage. 
cardo completely separated from stipes. Labium (Fig. 9) subconical, preapically constricted and apically rounded, almost membranous except in sclerotized area (pmsc); labial palpus (IbP) vestigial, lobe-like. Premental sclerite (pmsc) distinctly dilated, "Y" shaped, with 1 pair of sensil- la. Ligulate area with 1 tiny seta (lgs). Prementum (Prmt) with 1 seta (prms); postmentum (Pmt) with 3 setae (pms) laterally, pms2 (located medially) much longer than pms3 (located apically) which is somewhat longer than pms 1 (located basally).
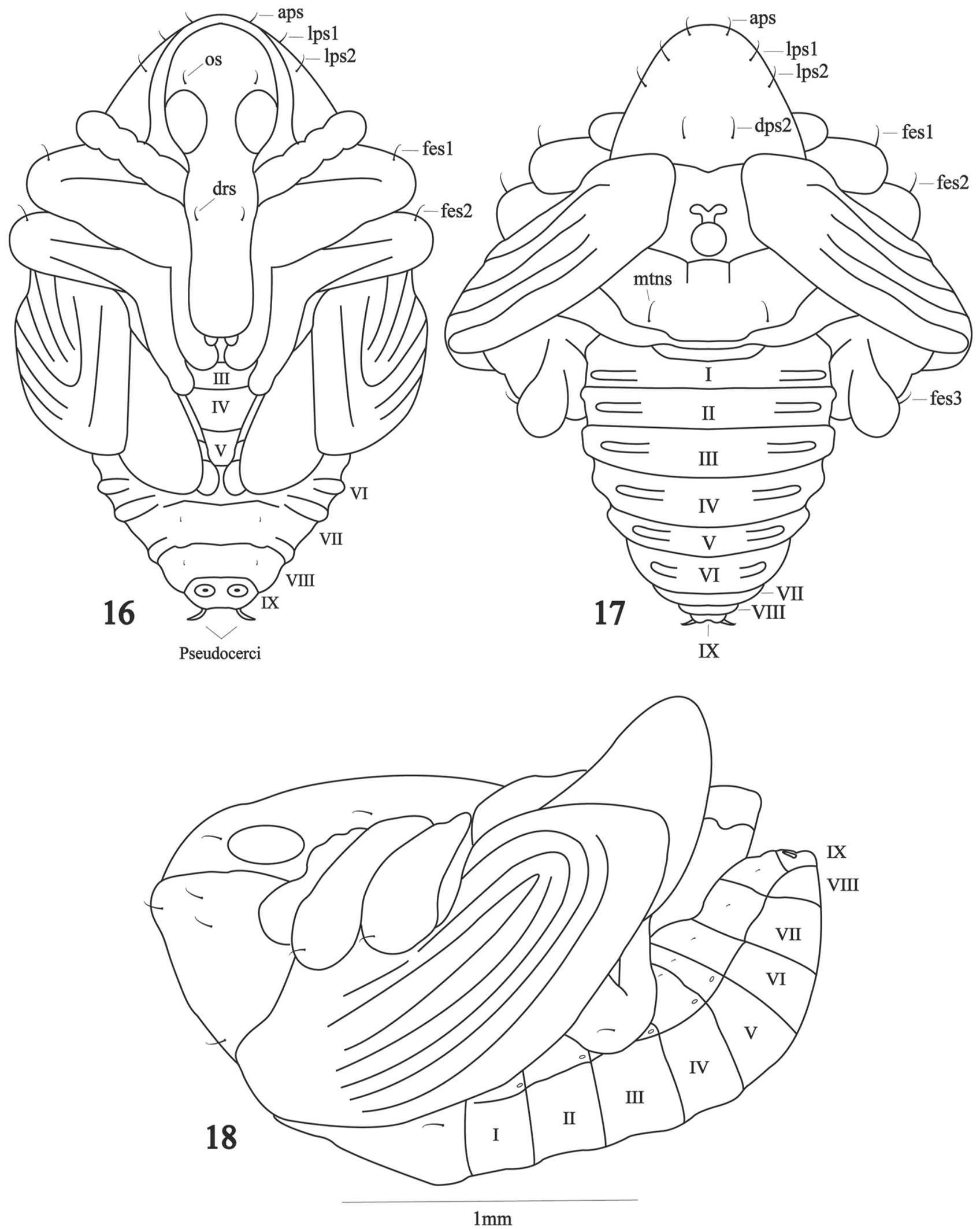

Figures 16-18. Apion brevicorne Gerstaecker, female pupal habitus: (16) ventral; (17) dorsal; (18) lateral. Abbreviations: [seta (ae)-s]: aps = apical pronotal s.; dps = discal pronotal s.; $d r s=$ distirostral s.; $d p s=$ discal pronotal s.; fes $=$ femoral s.; $/ p s=$ lateral pronotal s.; $m$ tns $=$ metanotal s.; $0 s=$ orbital s. 
Thorax (Fig. 1): Pronotal shield simple, without fold, unsclerotized; meso- and metanotum each with 2 folds (prodorsum and postdorsum). Spiracle (Fig. 15) laterally intersegmental between pro- and mesothorax, bicameral. Prothoracic epipleura indistinct, meso- and metathoracic epipleura distinct, not centrally tuberculate, without

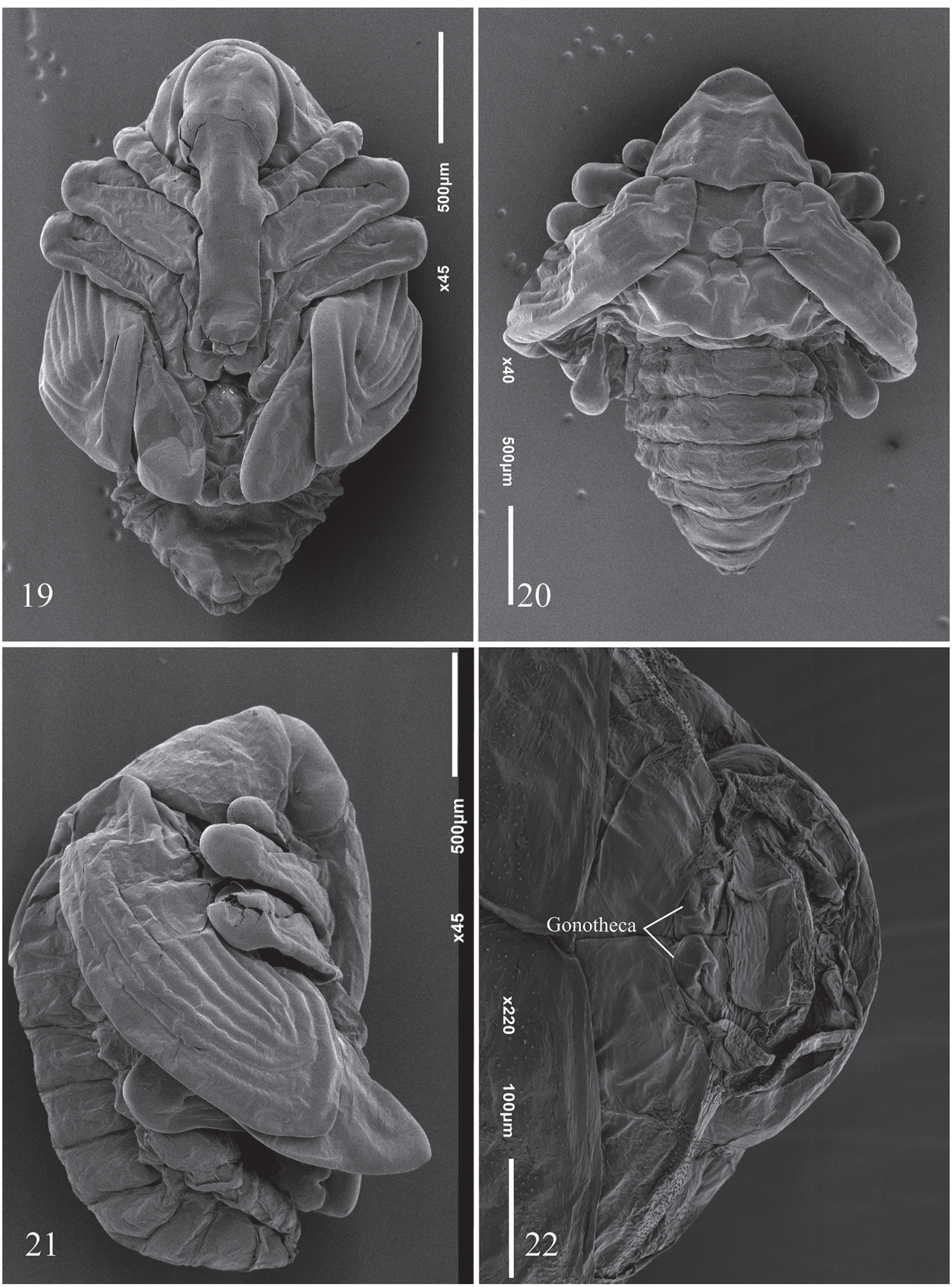

Figures 19-22. Apion brevicorne Gerstaecker, SEM micrographs pupa: (19-21) habitus: (19) ventral; (20) dorsal; (21) lateral; (22) female, abdominal segments VII-IX, ventral. 

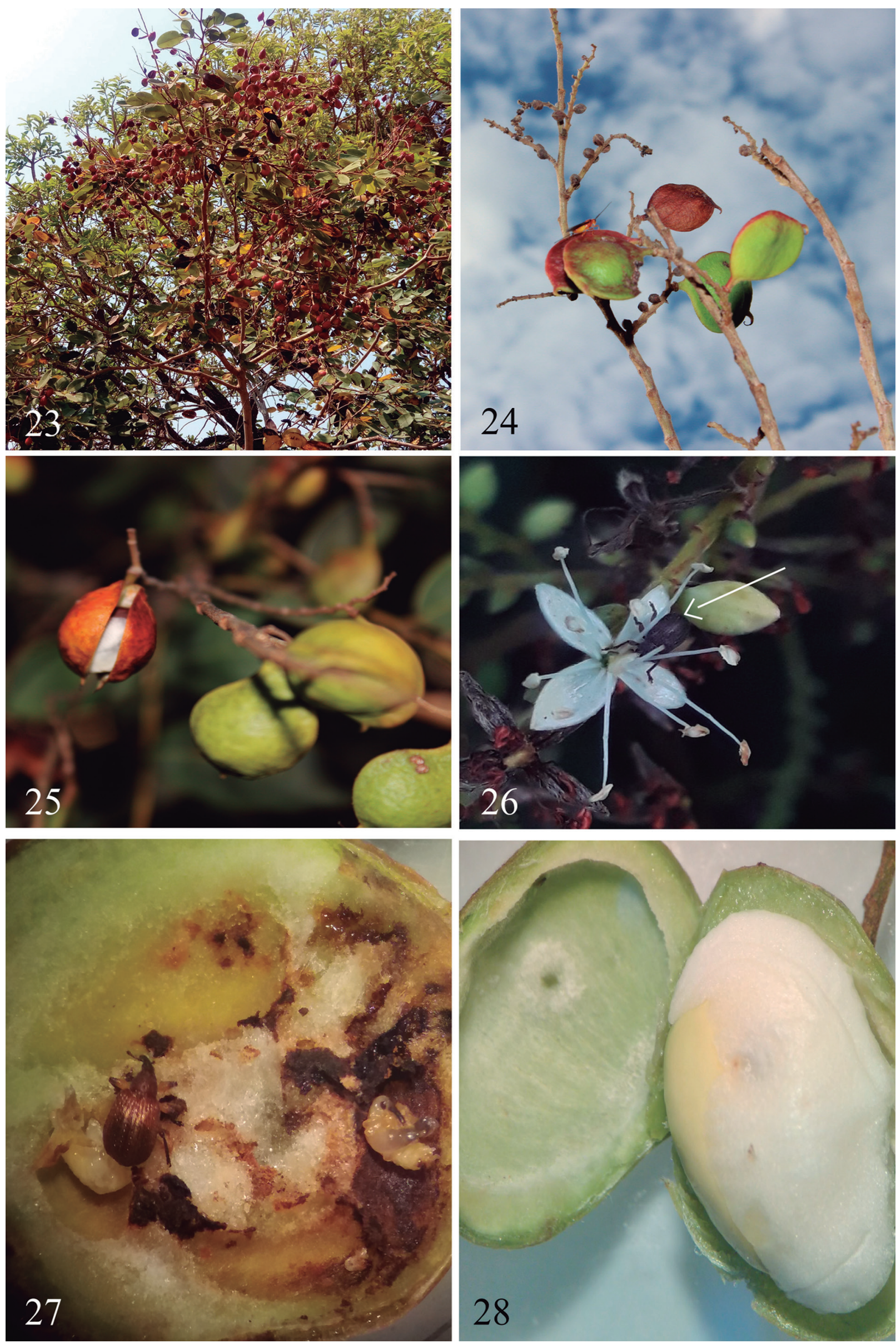

Figures 23-28. Copaifera malmei host plant of the Apion brevicorne: (23) host plant habitus; (24) fruit in intermediate stages of development; (25) mature fruit in dehiscence stage; (26) A. brevicorne feeding on floral nectar; (27) attacked fruit and (28) healthy fruit. 
setae. Pedal area defined, pedal lobe (papillae) present, 2-segmented. Pronotum with 2 setae (pns 1-2), transversally aligned near to middle area; meso- and metanotum each with an 1 prodorsum (prs) and 1 postdorsum (pds) setae, epipleurum without setae; pedal area with 3 setae (pda); sternum with 1 tiny seta.
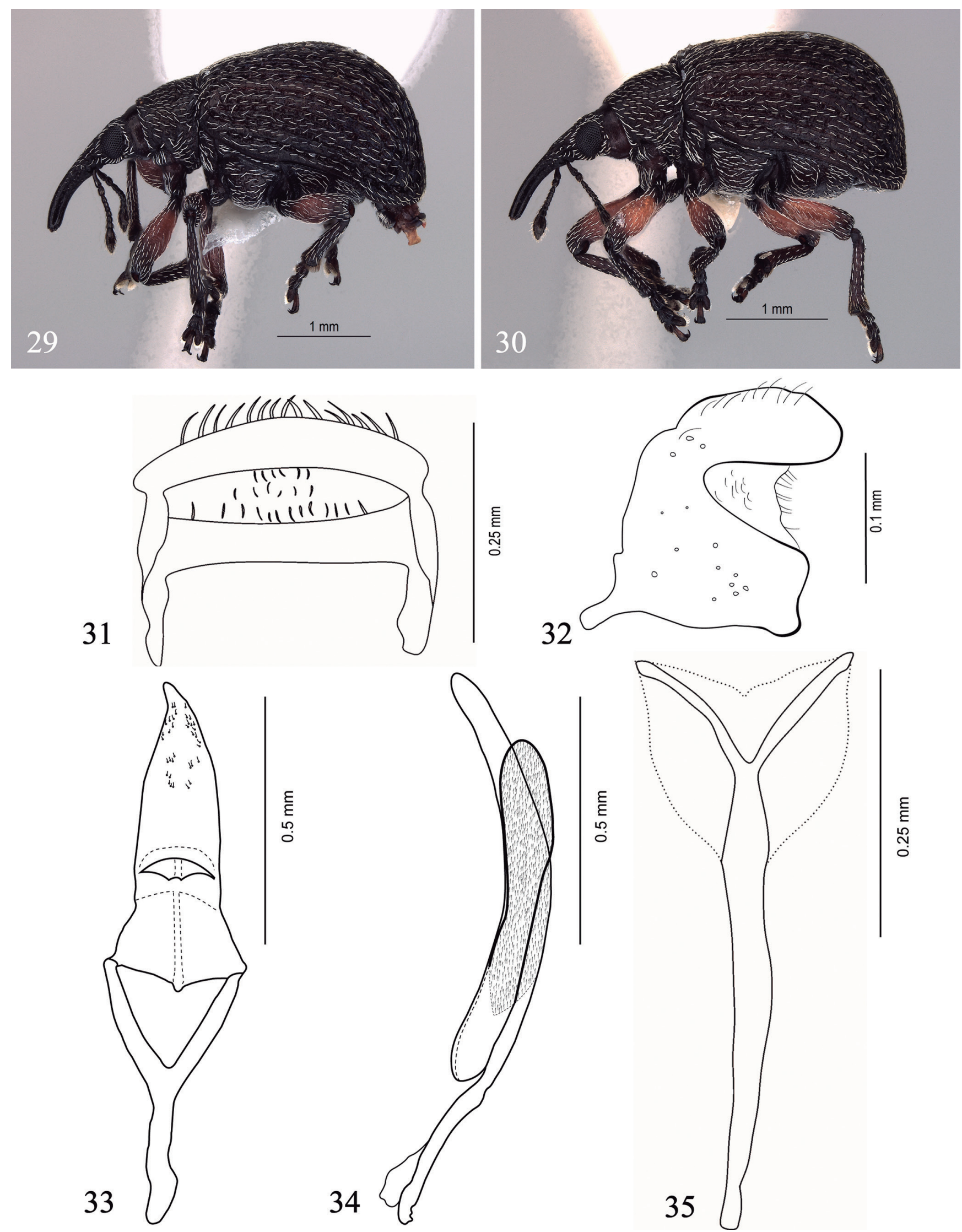

Figures 29-35. Apion brevicorne Gerstaecker: (29-30) habitus lateral, male, female; (31, 32) male pygidium, dorsal, lateral; (33) tegmen dorsal; (34) penis lateral; (35) spiculum gastrale. 
Table 1. Larval chaetotaxy (of one side of the body) compared among Neotropical Apion brevicorne and Palearctic species of Apioninae. Question marks indicate that setae were either not mentioned, not illustrated or doubtful in descriptions and figures. "Ind" indicates that the setae could not be counted because they are densely packed.

\begin{tabular}{|c|c|c|c|c|c|c|c|c|c|c|c|c|c|c|c|}
\hline \multirow[b]{2}{*}{ Body Part } & \multirow[b]{2}{*}{ Character } & \multicolumn{14}{|c|}{ Species } \\
\hline & & 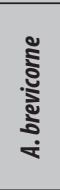 & 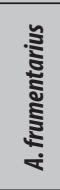 & ن & 悹 & 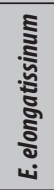 & 竎 & $\begin{array}{l}\overline{\mathbf{s}} \\
\text { ș } \\
\text { L }\end{array}$ & 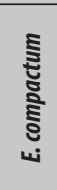 & 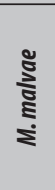 & 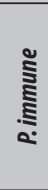 & 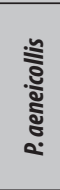 & 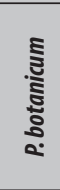 & 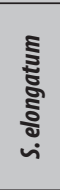 & 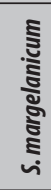 \\
\hline \multirow[t]{6}{*}{ Head } & posterior epicranial setae (pes) & 2 & 0 & $?$ & 4 & 4 & 4 & 4 & 4 & 0 & 0 & 0 & 6 & 4 & 0 \\
\hline & dorsal epicranial setae (des) & 3 & 5 & 4 & 4 & 4 & 4 & 4 & 4 & 4 & 5 & 3 & 5 & 5 & 3 \\
\hline & lateral epicranial setae (les) & 2 & 1 & 1 & 1 & 1 & 1 & 1 & 1 & 2 & 2 & 1 & 2 & 2 & 2 \\
\hline & frontal setae (fs) & 4 & 3 & 2 & 2 & 2 & 2 & 2 & 2 & $?$ & 3 & 3 & 4 & 5 & 3 \\
\hline & ventral cranial setae (Vcs) & 2 & 0 & 0 & 1 & 1 & 1 & 1 & 1 & $?$ & 2 & 0 & 2 & $?$ & 0 \\
\hline & clypeal setae (cls) & 1 & 1 & 1 & 1 & 1 & 1 & 1 & 1 & 2 & 1 & 1 & 1 & 1 & 1 \\
\hline \multirow[t]{13}{*}{ Mouthparts } & labral setae (Ims) & 3 & 3 & 1 & 1 & 1 & 1 & 1 & 1 & 1 & $?$ & 3 & 3 & 3 & 3 \\
\hline & anteromedian setae epipharynx (ams) & 2 & 3 & 2 & 2 & 2 & 2 & 2 & 2 & 3 & 2 & 2 & 2 & 3 & 3 \\
\hline & anterolateral setae epipharynx (als) & 2 & 4 & 3 & 2 & 2 & 2 & 2 & 2 & 2 & 3 & 2 & 2 & 3 & 3 \\
\hline & median epipharyngeal setae (mes) & 1 & 0 & 1 & 1 & 1 & 1 & 1 & 1 & 1 & 1 & 2 & 1 & 1 & 2 \\
\hline & mandibular setae (mds) & 2 & 1 & 2 & 2 & 2 & 2 & 2 & 2 & 2 & 2 & 1 & 1 & 2 & 0 \\
\hline & dorsal malar setae (dms) & Ind & 5 & 5 & 5 & 5 & 5 & 5 & 5 & 5 & 4 & 4 & 5 & 8 & 5 \\
\hline & ventral malar setae (vms) & Ind & 3 & $?$ & 3 & 4 & 4 & 4 & 4 & 1 & 1 & 3 & 4 & $?$ & 2 \\
\hline & stipetal setae (sts) & 2 & 3 & 1 & 1 & 1 & 1 & 1 & 1 & 2 & 2 & 1 & 1 & 1 & 3 \\
\hline & basioventral setae (mbs) & 1 & 1 & 1 & 1 & 1 & 1 & 1 & 1 & 0 & 0 & 1 & 0 & $?$ & 0 \\
\hline & palpiferal setae (pfs) & 2 & 0 & 2 & 2 & 2 & 2 & 2 & 2 & 2 & 2 & 0 & 2 & 2 & 0 \\
\hline & ligular setae (Igs) & 1 & 1 & 0 & 1 & 1 & 1 & 1 & 1 & 2 & 2 & 2 & 1 & 2 & 1 \\
\hline & premental setae (prms) & 1 & 0 & 1 & 1 & 1 & 1 & 1 & 1 & 1 & 1 & 1 & 1 & 1 & 1 \\
\hline & postmental setae (pms) & 3 & 3 & 3 & 3 & 3 & 3 & 3 & 3 & 3 & 2 & 3 & 3 & 2 & 3 \\
\hline \multirow[t]{9}{*}{ Thorax } & pronotal setae (pns) & 2 & 6 & 6 & 6 & 6 & 6 & 6 & 6 & 12 & 12 & 6 & 6 & 5 & 3 \\
\hline & prodorsal setae (prs) & 1 & 1 & 1 & 1 & 1 & 1 & 1 & 1 & 1 & 1 & 2 & 1 & 1 & 1 \\
\hline & postdorsal setae (pds) & 0 & 3 & 2 & 1 & 1 & 1 & 1 & 1 & 4 & 4 & 4 & 4 & 5 & 4 \\
\hline & alar setae (as) & 0 & 1 & 0 & 3 & 3 & 3 & 3 & 3 & 1 & 1 & 1 & 0 & 0 & 1 \\
\hline & spiracular setae (ss) & 0 & 0 & 2 & $?$ & $?$ & $?$ & $?$ & $?$ & 3 & 3 & 0 & 0 & 0 & 0 \\
\hline & epipleural setae (eps) & 0 & $2-3$ & $?$ & 1 & 1 & 1 & 1 & 1 & 1 & 1 & $2-3$ & 4 & 4 & $1-3$ \\
\hline & pleural setae (ps) & 0 & 1 & $?$ & 1 & 1 & 1 & 1 & 1 & 1 & 1 & 2 & 0 & 1 & 1 \\
\hline & pedal setae (pdas) & 3 & 4 & 2 & 2 & 2 & 2 & 2 & 2 & 2 & 2 & 6 & $3-4$ & 2 & 3 \\
\hline & sternal setae (sts) & 1 & 2 & 1 & 1 & 1 & 1 & 1 & 1 & 1 & 1 & 2 & 1 & 1 & 1 \\
\hline \multirow[t]{6}{*}{ Abdomen } & prodorsal setae (prs) & 1 & 1 & 1 & 1 & 1 & 1 & 1 & 1 & 1 & 1 & 2 & 1 & 1 & 1 \\
\hline & postdorsal setae (pds) & 1 & 2 & 2 & 2 & 2 & 2 & 2 & 2 & 4 & 4 & 5 & 6 & 5 & 3 \\
\hline & setae of spiracular area (ss) & 0 & 1 & 1 & 1 & 1 & 1 & 1 & 1 & 1 & 1 & 1 & 0 & 0 & 0 \\
\hline & epipleural setae (eps) & 0 & 1 & 1 & 1 & 1 & 1 & 1 & 1 & 1 & 1 & 2 & 0 & 1 & 1 \\
\hline & pleural setae (ps) & 0 & 1 & 1 & $?$ & $?$ & $?$ & $?$ & $?$ & 0 & 0 & 2 & 0 & 1 & 1 \\
\hline & sternal setae (sts) & 1 & $?$ & 1 & 1 & 1 & 1 & 1 & 1 & 0 & 0 & $?$ & 1 & 1 & $?$ \\
\hline
\end{tabular}

Abdomen (Fig. 1): Tergites I-VII, each with 2 folds, prodorsum with 1 prs, postdorsum with 1 pds; tergite VIII undivided with 2 setae; tergite IX undivided and reduced with 2 setae; with 6 pairs of subequal bicameral spiracles, each pair anterolaterally on tergites I-VI, VII and VII without spiracles; pleura I-VIII without setae, each sternum with 1 seta. Anal area with 2 lobes and 1 seta.

Pupal description (Figs. 16-21): Measurements (in $\mathrm{mm}$ ): body length: 3.00-4.13, body width (metathorax): 1.22-1.50.

General: Adecticous and exarate; body setae on tubercles, setae greatly reduced in number and size.

Colouration: Inner body pure white, except eyes, which are pale to dark.
Rostrum in ventral view (Figs. 16, 22): Apex extends past mesocoxae but not to metacoxae, mesorostrum dilated, mandibular theca projected; 1 distirostral seta (drs).

Head: Frons with 1 seta (os), similar in size as drs, behind eyes. Antennae basally near prosternum and apically extend to propleura, oblique to protibia.

Thorax: In dorsal view, pronotum with 1 apical pronotal seta (aps), 2 lateral pronotal setae (Ips 1-2), and 1 discal pronotal seta (dps2); in ventral view, aps and Ips 1-2 completely visible. Mesonotum without setae; metanotum with 1 seta (mtns). Legs (Figs. 16-21): in ventral view, metatibiae and femora covered by pterothecae, front and middle legs and metatarsomere visible; pro-, meso- and metafemora apically bearing 1 slightly out- 
Table 2. Pupal chaetotaxy (of one side of the body) compared among Neotropical Apion brevicorne and Palearctic species of Apioninae. Question marks indicate that setae were either not mentioned, not illustrated or doubtful in descriptions and figures.

\begin{tabular}{|c|c|c|c|c|c|c|c|c|c|c|}
\hline \multirow[b]{2}{*}{ Body Part } & \multirow[b]{2}{*}{ Character } & \multicolumn{9}{|c|}{ Species } \\
\hline & & 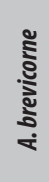 & ن & 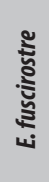 & 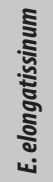 & 妾 & 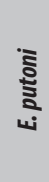 & 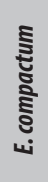 & 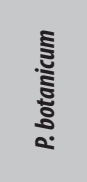 & 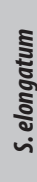 \\
\hline \multirow[t]{7}{*}{ Head } & basirostral setae (brs) & 0 & 0 & 1 & 1 & 1 & 2 & 2 & 0 & 0 \\
\hline & distirostral setae (drs) & 1 & 0 & 1 & 1 & 1 & 1 & 1 & 1 & 1 \\
\hline & rostral lateral setae (r/s) & 0 & 0 & 0 & 0 & 0 & 0 & 0 & 0 & 0 \\
\hline & orbital setae (os) & 0 & 1 & 1 & 1 & 1 & 0 & 1 & 1 & 0 \\
\hline & postorbital setae (pos) & 0 & 0 & 0 & 0 & 0 & 1 & 0 & 0 & 0 \\
\hline & supraorbital setae (sos) & 1 & 0 & 0 & 0 & 0 & 1 & 0 & 0 & 1 \\
\hline & vertical setae (vs) & 0 & 0 & 1 & 1 & 1 & 1 & 1 & 0 & 0 \\
\hline \multirow[t]{6}{*}{ Thorax } & apical pronotal setae (aps) & 1 & 2 & 1 & 2 & 1 & 1 & 1 & 2 & 2 \\
\hline & basal pronotal setae (bps) & 0 & 0 & 1 & 1 & 1 & 1 & 1 & 1 & 1 \\
\hline & discal pronotal setae (dps) & 1 & 1 & 1 & 1 & 1 & 1 & 2 & 2 & 1 \\
\hline & lateral pronotal setae (Ips) & 2 & 1 & 2 & 1 & 2 & 2 & 2 & 1 & 1 \\
\hline & mesonotal setae (msns) & 0 & $?$ & 1 & 1 & 1 & 1 & 1 & 0 & 0 \\
\hline & metanotal setae (mtns) & 1 & $?$ & 2 & 4 & 2 & 2 & 2 & 2 & 3 \\
\hline Legs & femoral setae (fes) & $1-3$ & $1-3$ & $1-3$ & $1-3$ & $1-3$ & $1-3$ & 3 & 1 and 3 & 1 \\
\hline \multirow[t]{3}{*}{ Abdomen } & postdorsal setae (pds) & 0 & $?$ & 2 & 2 & 2 & 3 & 3 & $3-4$ & 7 \\
\hline & pleural setae & 0 & $?$ & 1 & 1 & 1 & 1 & 1 & $?$ & 0 \\
\hline & ventral setae & $0-1$ & $?$ & 2 & 2 & 2 & 2 & 1 & 2 & 0 \\
\hline
\end{tabular}

curved seta (fes), each seta inserted in a small rounded protuberance.

Abdomen: In ventral view, ventrites IV-IX visible (Fig. 16), in dorsal view tergites I-VII about equal in length and decreasing in width (Fig. 17), segments VII-IX clearly and gradually reduced; 5 spiracles present, positioned on pleura I-V, bicameral (Fig. 18); pseudocerci inconspicuous at segment IX, unpigmented; in ventral view, ventrites IV-VIII with 1 pair of setae; in dorsal view, tergites I-IX without setae.

Sexual dimorphism: Female with small round convexity on each side of sternum on ninth abdominal segment (Figs. 16, 22).

Biological information (Figs. 23-28): Apion brevicorne is associated with C. malmei. Adults may feed on nectar in flowers at any time, day or night. Copulation and oviposition occur on fruits at any stage of development. The oviposition is endophytic and eggs are easily found only in fruits at an early stage of development. As in most weevils, the damage caused by A. brevicorne to the seed is mostly by larvae that develop within the seed, where they feed on developing endocarp. Pupae were always found within seeds (without a pupal chamber) along with feces that indicate that the larvae consumed the entire endocarp, and so development is completed within the seed. The number of adults in each seed is quite variable. Seed pods that contain larvae, pupae or adults show no external evidence of presence. This suggests that for adults to disperse, they depend on the dry dehiscence of the fruit. Dead adults and pupae can be found within dry seed pods that did not dehisce.

\section{DISCUSSION}

All of the 13 species of Apioninae whose immature stages have been described are in seven of the subtribes of the Apionini (Williams, 1968; May, 1994; Sanz Benito \& Gurrea Sanz, 1999; Nikulina, 2016; Wang et al., 2013; Letowski et al., 2015). We found that morphology of Apion brevicorne larvae and pupae described herein are very different from other species in these subtribes (especially the reduced chaetotaxy of the mala, thorax and abdomen) and different from other generalized endophagous Apioninae (May, 1993, 1994; Wang et al., 2013; AlonsoZarazaga \& Wanat, 2014) and other Curculionoidea (Oberprieler et al., 2014) (Tables 1 and 2). Establishing homologies for the reduced number of setae for the Apioninae is complicated because of the various usages of nomenclature for the Curculionoidea (Anderson, 1947, Marvaldi, 1999; Wang et al., 2013). Differences in terminology and abbreviations among publications can lead to errors or inconsistencies in morphology, phylogeny and interpretation (Lira et al., 2017).

Larval characteristics that vary among the Apioninae and which can be used to identify genera and species of Apionini are mainly chaetotaxy of the head and mouthparts. Variability is common interspecifically as well as intrageneric and thus may be used to diagnose species (Sanz Benito \& Gurrea Sanz, 1999; Wang et al., 2013; Alonso-Zarazaga \& Wanat, 2014).

The indistinct frontal line and endocarina we describe for Apion brevicorne is the first observation of these characters in Apioninae. The frontal line may be absent in some groups of Curculionoidea (Oberprieler et al., 2014) but is always present in Apioninae (Alonso-Zarazaga \& 
Wanat, 2014). This may be very useful in future phylogenetic analyses of Apioninae.

The hypopharyngeal bracon, a bar that connects the two hypopharyngeal margins and which supports the hypopharynx, separates larvae of the Curculionoideae from those of the Bruchidae and Chrysomelidae (Lawrence, 1991; Oberprieler et al., 2014). The hypopharyngeal bracon is present and distinct in the primitive Australo-Pacific Neocyba sp. (Rhadinocybitae) (May, 1993) and absent in A. brevicorne. Its absence in Apioninae was first noted in P. botanicum (Wang et al., 2013), perhaps because it was uncommon to refer to ventral characters of the epicranium when describing apionine larvae (Bennett, 1992; Emden, 1938; Gosik et al., 2010; May, 1994; Sanz Benito \& Gurrea Sanz, 1999). More apionine species need to be studied to determine the importance of the hypopharyngeal bracon as a diagnostic character (Wang et al., 2013).

The number of spiracles (6 pairs) in larval A. brevicorne and Holartic Podapion Riley, 1883 differ from that for typical Apioninae (7 pairs) as well (Emden, 1938; Scherf, 1964; Wang et al., 2013; Alonso-Zarazaga \& Wanat, 2014; Letowski et al., 2015). Also, pupal A. brevicorne have less setae everywhere except on the femora. Pupal chaetotaxy ranges from 6 setae on the head and rostrum of Exapion putoni (Sanz Benito \& Gurrea Sanz, 1999) to one in Exapion ulisis (May, 1994). Thus, pupal chaetotaxy can be used to identify pupae in other subtribes or genera of the Apionini (May, 1994; Sanz Benito \& Gurrea Sanz, 1999; Wang et al., 2013).

In the female pupae, the gonotheca of the ninth abdominal sternum is divided into two parts. This pattern of sexual dimorphism seems to be common in pupae in a variety of Curculionoidea (Burke, 1968; May, 1994; Sarro et al., 2004; De Sousa et al., 2004), but has never been noted in the Apioninae (May, 1994; Sanz Benito \& Gurrea Sanz, 1999; Wang et al., 2013).

Morphological and taxonomic studies of immature stages of Apioninae are still uncommon, yet such studies are fundamental for species identification, as well as for understanding their taxonomy and systematics. Apioninae biology is also poorly known in the Neotropics. Now, with our results, we were able to identify pests of unidentified genera of Neotropical Apioninae (Apion sp.) that are found on three species of Copaifera: C. sabulicola J. Costa \& L.P. Queiroz, Copaifera luetzelburgii Harms and Copaifera depilis Dwyer (Santos et al., 2015). We confirmed that samples of these thitherto unknown species also pertain to $A$. brevicorne (Figs. 29-35). Thus, A. brevicorne is oligophagous in contrast with other Brazilian Apioninae in their association with several Copaifera spp. Pests of Copaifera spp. may cause reduced productivity, with important detrimental effects on plant populations (Santos et al., 2015). Studies should continue using integrative taxonomy to determine the number of species of Copaifera that may host A. brevicorne, whether each host species is associated with additional taxonomic units of the weevil, and the taxonomic position of the species as a possible new genus within the Apionini.

\section{ACKNOWLEDGMENTS}

We are indebted to the Fundação de Amparo à Pesquisa do Estado de Mato Grosso (FAPEMAT) for research grants in support of the first and second authors. We thank Dr. Jorge Antônio Silva Costa (Universidade Federal do Sul da Bahia) and Dr. Erica Pereira de Campo (Universidade Federal de Mato Grosso, Campus de Rondonópolis) for the identification of the host plant of the apionine studied. James J. Roper, Ph.D., revised the English text in its entirety. We also thank Dr. Cleide Costa, Museu de Zoologia, Universidade de São Paulo, who encouraged the study and production of knowledge about beetles in Brazil.

\section{REFERENCES}

Alonso-Zarazaga, M.A. 1990. Revision of the supraspecific taxa in the Palaearctic Apionidae Schoenherr, 1823 (Coleoptera, Curculionoidea). 2. Subfamily Apioninae Schoenherr, 1823: Introduction, keys and descriptions. Graellsia, 46: 19-156.

Alonso-Zarazaga, M.A. 2004. Apionidae (Coleoptera). In: Bousquets, J.L. \& Morrone, J.J. (Eds.). Biodiversidad, taxonomía y biogeografía de artrópodos de México: hacia una síntesis de su conocimiento, México, D.F., Talleres de Jiménez Editores e Impresores. v. 4, p. 691-700.

Alonso-Zarazaga, M. \& Wanat, M. 2014. Apioninae Schoenherr, 1823. In: Leschen, R.A.B. \& Beutel, R.G. (Eds.). Coleoptera, Beetles. Vol. 3: Morphology and systematics: Phytophaga. Berlin, Walter de Gruyter. p. 395-415. (Handbook of Zoology, Arthropoda: Insecta)

Alonso-Zarazaga, M.A. \& Lyal, C.H.C. 1999. A world Catalogue of Families and Genera of Curculionoidea (Insecta: Coleoptera) (Excepting Scolytidae and Platypodidae). Barcelona, Entomopraxis, S.C.P. Edition. 315p.

Anderson, R.S. \& Kissinger, D.G. 2002. Family 129. Brentidae Billberg 1820. In: Arnett, R.H.; Thomas, M.; Skelley, P.E. \& Frank, J.H. (Eds.). American Beetles. Boca Raton, Florida, CRC Press. v. 2, p. 711-719.

Anderson, W.H. 1947. A terminology for the anatomical characters useful in the taxonomy of weevil larvae. Proceedings of the Entomological Society of Washington, 49: 123-132.

Badenes-Perez, F.R. \& Jhonson, M.T. 2007. Ecology and impact of Allorhogas sp. (Hymenoptera: Braconidae) and Apion sp. (Coleoptera: Curculionoidea) on fruits of Miconia calvescens DC (Melastomataceae) in Brazil. Biological Control, 43: 317-322.

Bennett, A.L. 1992. A contribution to the morphology of Apion soleatum Wagner (Coleoptera: Apionidae), a pest of cotton. The larva and pupa, including a discussion of spiracular form. Journal of the Entomological Society of Southern Africa, 55: 185-195.

Bouchard, P.; Bousquet, Y.; Davies, A.E.; Alonso-Zarazaga, M.A.; Lawrence, J.F.; Lyal, C.H.; Newton, A.F.; Reid, C.A.; Schmitt, M. \& Smith, A.B. 2011. Family-group names in Coleoptera (Insecta). Zookeys, 88: 1-972.

Burke, H. 1968. Pupae of the weevil tribe Anthonomini (Coleoptera: (urculionidae). Technical Monographs Texas Agricultural Experiment Station, 5: 1-92.

De Sousa, W.0. \& Ribeiro-Costa, C.S. 2018. Two new Brazilian species of Neapion Alonso-Zarazaga, 1990, subgenus Neotropion Alonso-Zarazaga, 1990 with a key to South American species (Brentidae: Apioninae). Zootaxa, 4402(3): 551-562.

De Sousa, W.0;; Ribeiro-Costa, C.S. \& Rosado-Neto, G.H. 2019. A preliminary overview of the Brazilian Apioninae (Coleoptera: Brentidae) with an illustrated key for genera, and a checklist with distribution information. Biota Neotropica, 19(4): 1-18. e20190813; 
De Sousa, W.O.; Rosado-Neto, G.H.; Moreira, M.A.B. \& Zarbin, P.H.G. 2004. Description of the larva and pupa of the papaw borer weevil Pseudopiazurus papayanus (Marshall) (Coleoptera, Curculionidae, Piazurini). Revista Brasileira de Entomologia, 48(2): 331-334.

Emden, F.V. 1938. On the Taxonomy of Rhynchophora Larvae (Coleoptera). Transactions of the Royal Entomological Society of London, 87: 1-37.

Gosik, R.; Letowski, J. \& Kozak, E. 2010. Morphology of the mature larvae and pupa of Diplapion confluens (Kirby, 1808) (Coleoptera: Apionidae). Polish Journal of Entomology, 79(3): 211-221.

Kissinger, D.G. 1968. Curculionidae subfamily Apioninae of North and Central America with reviews of the world genera of Apioninae and world subgenera of Apion Herbst (Coleoptera). South Lancaster, Mass.,Taxonomic Publications. 559p.

Kissinger, D.G. 2002. A New Genus of Apionidae from Chile and Argentina. The Coleopterists Bulletin, 56(2): 315-316.

Kissinger, D.G. 2003. A new species of Coelocephalapion Wagner (Apionidae) from Venezuela with host Hyptis suaveolens (L.)Poit. (Lamiaceae). The Coleopterists Bulletin, 57(1): 99-104.

Kissinger, D.G. 2005a. Review of Apioninae of Chile (Coleoptera: (urculionoidea: Apionidae). The Coleopterists Bulletin, 59(1): 71-90.

Kissinger, D.G. 2005b. A New Species of Coelocephalapion Wagner (Coleoptera: Curculionoidea: Apionidae: Apioninae) from Argentina and Chile Associated with the Genus Prosopis L. (Fabaceae). The Coleopterists Bulletin, 59(4): 493-500.

Kuschel, G. 1995. A phylogenetic classification of Curculionoidea to families and subfamilies. Memoir of the Entomological Society of Washington, 14: $5-33$.

Lawrence, J.F. 1991. Order Coleoptera. 34. In: Stehr, F.W. (Ed). Immature Insects. Dubuque, lowa, Kendall Hunt Pub. Co. v. 2, p. 144-298.

Letowski, J.; Pawlega, K.; Scibior, R. \& Rojek, K. 2015. The morphology of the preimaginal stages of Squamapion elongatum (Germar, 1817) (Coleoptera, Curculionoidea, Apionidae) and notes on its biology. ZooKeys, 519: 101-115.

Lima, E.C.; Paiva, R.; Nogueira, R.C.; Soares, F.P.; Emrich, E.B. \& Silva, A.A.N. 2008. Callus induction in leaf segments of Croton urucurana Baill. Ciência e Agrotecnologia, 32(1): 17-22.

Lira, A.O.; Rosado-Neto, G.H.; Marques, M.I. \& De Sousa, W.0. 2017. First description of the larva and pupa of the coconut palm borer Homalinotus depressus (Linnaeus, 1758) (Coleoptera: Curculionidae: Molytinae) and a discussion about the terminology for immature forms. Zootaxa, 4311(4): 589-599.

Maia, V.C. 2012. Coleopterous galls from the Neotropical region. Papéis Avulsos de Zoologia, 52(15): 175-184.

Marvaldi, A.F. 1999. Larval morphology in Curculionidae (Insecta: Coleoptera). Morfologia larval en Curculionidae (Insecta: Coleoptera). Acta Zoologica Lilloana, 45: 7-24.

Marvaldi, A.E.; Sequeira, A.S.; O'Brien, C.W. \& Farrell, B.D. 2002. Molecular and morphological phylogenetics of weevils (Coleoptera: Curculionoidea): do niche shifts accompany diversification? Systematic Biology, 51(5): 761-785.
May, B.M. 1993. Larvae of Curculionoidea (Insecta: Coleoptera): a systematic overview. Fauna of New Zealand, 28: 1-221.

May, B.M. 1994. An introduction to the immature stages of Australian Curculionoidea. In: Australian weevils. (Coleoptera: Curculionoidea). Volume 2: Brentidae, Eurhynchidae, Apionidae and a chapter on immature stages. Melbourne, CSIRO. p. 365-726.

Nikulina, 0.N. 2016. Morphology of Larvae of the Weevils Apion frumentarium, Pseudaplemonus aeneicollis, and Stenopterapion margelanicum (Coleoptera, Apionidae). Entomological Review, 95(1): 65-73.

Oberprieler, R.G.; Marvaldi, A.E. \& Anderson, R.S. 2007. Weevils, weevils, weevils everywhere. Zootaxa, 1668: 491-520.

Oberprieler, R.G.; Andreson, R.S. \& Marvaldi, A.E. 2014. Curculionidea Latreille 1802. Introduction, phylogeny. In: Leschen, R.A.B. \& Beutel, R.G. (Eds.). Coleoptera, Beetles. Vol. 3: Morphology and systematics: Phytophaga. Berlin, Walter de Gruyter. p. 285-300. (Handbook of Zoology, Arthropoda: Insecta)

Santos, I.M.; Costa, J.A.S.; Costa, C.B.N. \& Calado, D. 2015. Predação de sementes por insetos em três espécies simpátricas de Copaifera L. (Fabaceae). Biotemas, 28: 87-95.

Sanz Benito, M.J. \& Gurrea Sanz, P. 1999. Immature stages of five species of the genus Exapion Bedel (Coleoptera: Brentidae, Apioninae) associated with the seeds of Genista (Tournfourt) and Cytisus L. (Fabaceae). Coleopterists Bulletin, 53: 8-26.

Sarro, F.B.; Crocomo, W.B. \& Ferreira, J.M.S. 2004. Aspectos da biologia e morfologia da broca do pedúnculo floral do coqueiro, Homalinotus coriaceus (Gyllenhal) (Coleoptera: Curculionidae). Neotropical Entomology, 33(1): 7-12.

Scherf, H. 1964. "Die Entwicklungsstadien der mitteleuropäischen Curculioniden (Morphologie, Bionomie, Ökologie)". Abhandlungen der Senckenbergischen Naturforschenden Gesellschaft, 506: 1-335.

Wanat, M. 2001. Genera of Australo-Pacific Rhadinocybinae and Myrmacicelinae with biogeography of the Apionidae (Coleoptera: Curculionoidea) and phylogeny of the Brentidae (s. lato). Mantis, Olsztyn. 432p.

Wang, Z.; Alonso-Zarazaga, M.A.; Zhou, D. \& Zhang, R. 2013. A description of preimaginal stages of Pseudaspidapion botanicum Alonso-Zarazaga \& Wang, 2011 (Apionidae, Curculionoidea). ZooKeys, 260: 49-59.

Wibmer, G.J. \& O'Brien, C.W. 1986. Annotated checklist of the weevils (Curculionidae sensu lato) of South America (Coleoptera: Curculionoidea). Memoirs of the American Entomological Institute, 39: 1-563.

Williams, P. 1968. The Larvae of Apion immune Kirby and Apion malvae (F.) (Coleoptera: Curculionidae). The Proceeding of the Royal Entomological Society London, 43(1-3): 21-26.

Winter, S.; Friedman, A.L.; Astrin, J.J.; Gottsberger, B. \& Letsch, H. 2017. Timing and host plant associations in the evolution of the weevil tribe Apionini (Apioninae, Brentidae, Curculionoidea, Coleoptera) indicate an ancient co-diversification pattern of beetles and flowering plants. Molecular Phylogenetics and Evololution, 107: 179-190. 